\title{
Zum Gedenken an Reinhold Brinkmann (1934-2010)
}

von Christoph Wolff (Cambridge, Massachusetts)

Wer immer Reinhold Brinkmann in den letzten Monaten seiner schweren und physisch paralysierenden Erkrankung begegnete, konnte nicht anders als tief beeindruckt sein von der Wachheit seines Geistes, seinem ungebrochenen Interesse am Weltgeschehen und seinem Verlangen, das Aktuellste aus Musikleben und Musikwissenschaft zu erfahren. Er war ein ausgesprochener Gegenwartsmensch, dem Rückblicke nur wenig bedeuteten - es sei denn, es handelte sich um Geschichte, aus der sich Lehren ziehen ließen. Nun aber denken wir an ihn zurück. Er starb am 10. Oktober 2010 in Eckernförde, der Heimatstadt seiner Frau Dorothea Brinkmann, die ihr Leben nahezu ein halbes Jahrhundert mit dem seinigen teilen konnte.

Reinhold Brinkmann wurde am 21. August 1934 im oldenburgischen Wildeshausen geboren. Er erinnerte gern daran, dass das kleine Städtchen seinerzeit auch der Geburtsort des Hamburger Organisten und Komponisten Johann Adam Reincken war. Wichtiger war ihm freilich, dass sein Vater in den 1920er Jahren dort als einer der letzten Stadtmusiker noch eine echte Stadtpfeifer-Ausbildung in allen Instrumentenkategorien genossen hatte. So wurde Brinkmann von Kindertagen an von einer praktisch-musikalischen Umgebung geprägt, die ihn wie auch seinen Bruder dazu brachte, sich beruflich der Musik zuzuwenden. Er studierte 1955-61 Schulmusik und Germanistik in Hamburg an der Musikhochschule (Hauptfach Klavier) und Universität und wechselte danach mit einem Stipendium der Studienstiftung zur wissenschaftlichen Vertiefung seiner Ausbildung nach Freiburg im Breisgau zu Hans-Heinrich Eggebrecht, Walter Rehm und Friedrich Maurer.

Der wissenschaftliche Schwerpunkt Eggebrechts lag damals ausschließlich auf dem Mittelalter und der älteren Musik, was Brinkmann - seiner Herkunft treu - begeistert aufgriff. Doch seit er auf einem Ferienkurs der Studienstiftung in einem Konzert des amerikanischen Pianisten Paul Jacobs das gesamte Klavierwerk Schönberg und dazu Klavierstücke von Stockhausen gehört hatte, erwachte bei ihm ein starkes Interesse, sich der Musik der Zweiten Wiener Schule auch forschend zu nähern. Da jedoch Neue Musik weder in Freiburg noch andernorts damals wissenschaftlich behandelt wurde, oblag es ihm, sich Stoff und Methode gleichsam autodidaktisch zu erarbeiten. Er legte dann mit einer Dissertation über Arnold Schönberg, Drei Klavierstücke op. 11: Studien zur frühen Atonalität bei Schönberg (als Band 7 der „Beihefte zum Archiv für Musikwissenschaft" 1969 erschienen) eine bahnbrechende Arbeit vor - bahnbrechend in zweifachem Sinne: zum einen als analytische Studie zu einem Schlüsselwerk der Dodekaphonie (noch nach mehr als dreißig Jahren aktuell, wie der Nachdruck aus dem Jahr 2000 mit neuem Vorwort belegt); sodann als Anstoß für seinen akademischen Lehrer Eggebrecht, sich der Moderne zuzuwenden.

Reinhold Brinkmann wurde nach seiner Promotion 1967 der erste Assistent des im gleichen Jahr auf den Lehrstuhl an der Freien Universität Berlin berufenen Rudolf Stephan, der wie auch Carl Dahlhaus einen großen und bleibenden Einfluss auf den 
jungen Kollegen ausübte. Nach der Habilitation 1970 im damaligen West-Berlin nahm Brinkmann 1972 einen Ruf auf das Ordinariat der Universität Marburg an; von dort aus leitete er bis 1980 zugleich das Institut für Neue Musik und Musikerziehung in Darmstadt. 1980 kehrte Brinkmann nach (West-|Berlin zurück, jedoch an die Hochschule für Musik, heute Teil der Universität der Künste. 1984 erreichte ihn dann ein Ruf der Harvard University, dem er ein Jahr später folgte. Er lehrte bis zu seiner Emeritierung 2003 für 18 Jahre lang (seit 1990 als James Edward Ditson Professor) in Cambridge/Mass., ab 1996 in den Sommermonaten zusätzlich in Berlin als Honorarprofessor der HumboldtUniversität.

Als akademischer Lehrer wandte sich Brinkmann mit pädagogischer Leidenschaft und lebendigem Forschergeist vor allem der Musikgeschichte, -theorie und -ästhetik der letzten beiden Jahrhunderte zu. Seine zahlreichen und die verschiedensten musikalischen Gattungen durchquerenden Veröffentlichungen spiegeln das breite Spektrum von Beethoven über Schubert, Schumann, Wagner und Brahms bis zu Schönberg, Webern, Scriabin, Varèse, Eisler und Rihm. Besonders faszinieren die immer wieder von ihm eingeflochtenen Ausblicke in die Kunst- und Literaturgeschichte, besonders prägnant etwa in dem Aufsatz "On the Problem of Establishing ,Jugendstil' as a Category in the History of Music: With a Negative Plea" oder in der Monographie Schumann und Eichendorf: Studien zum Liederkreis Op. 39 (1979). Brinkmanns musikalische Analysen sind immer auch quellenkritisch untermauert. Nicht zuletzt dokumentiert seine Mitwirkung an der Schönberg-Gesamtausgabe (u. a. mit der Edition von Pierrot lunaire) seine starke Bindung an philologische Prinzipien.

Über allem entscheidend war für Brinkmann freilich der beständige Dialog mit den Akteuren der Neuen Musik, darunter anfänglich vor allem Dieter Schnebel. Welch produktiven Stellenwert ein solcher Dialog für beide Seiten haben konnte, erläutert das 2001 erschienene, immerhin 150 Seiten umfassende Büchlein musik nachdenken Reinhold Brinkmann und Wolfgang Rihm im Gespräch. Harvard bot ihm seinerzeit den direkten und beständigen Anlass zum Dialog im Kontakt mit den Komponisten-Kollegen im Music Department: Earl Kim und Leon Kirchner (beide Schönberg-Schüler aus der Zeit in Los Angeles), Mario Davidovsky und Bernard Rands sowie - für die beiden Serien der Norton Lectures - John Cage und Luciano Berio.

Kein in den USA arbeitender Deutscher kann sich den Aus- und Nachwirkungen der nationalsozialistischen Gewaltherrschaft und des Holocaust entziehen, zumal sie sich im Universitätsbereich durch den Umgang mit Kollegen und Studenten fast täglich manifestieren. Das Thema der Musiker-Vertreibungen und ihrer Konsequenzen stellt sich in diesem Umfeld von selbst. Brinkmann hat es gemeinsam mit Christoph Wolff in der Form eines Harvard-Symposiums aufgegriffen und 1999 in einem umfassenden Bericht vorgelegt: ,Driven into Paradise': the Musical Migration from Nazi Germany to the United States.

In die amerikanische Universitäts-Szene und Musikwelt hat sich Brinkmann sehr rasch eingefügt und konnte die starke Resonanz seines persönlichen und wissenschaftlichen Engagements nach seiner Emeritierung noch deutlich erleben und spüren. Vor allem konnte er sich noch daran erfreuen, wie sein offenes und kritisches Herangehen an die Texte, Klänge und Inhalte neuer und neuester Musik einen frischen und sicher- 
lich nachhaltigen Akzent im transatlantischen Verständnis der musikalischen Moderne gesetzt hatten. Das erschien ihm letztlich sogar wichtiger als seine Ehrung mit dem renommierten Ernst von Siemens Musikpreis, der ihm 2001 in München als zweitem Musikwissenschaftler (nach H. R. Robbins Landon, 1991) verliehen wurde.

In einem Interview nach dem Münchner Festakt fragte ihn der Journalist Max Nyffeler: „Was wünschen Sie Ihrem Fach für die Zukunft?" Reinhold Brinkmanns spontane Antwort: „Das ist eine schwere Frage. Ich wünsche ihm einen ganz offenen und kritischen Nachwuchs, der die Dinge tut, die ich nicht mehr schaffe oder die ich aus Gründen meiner Konstitution oder meiner Beengtheit nicht gemacht habe. Ein Fach, das sehr weltoffen ist und sich sehr aus der Nähe zur künstlerischen Produktion der Gegenwart entwickelt." Er hat damals wohl kaum daran gedacht, dass in dieser Antwort ein Vermächtnis beschlossen liegen könnte.

\section{Dietrich Berke zum Gedenken}

Am 16. Oktober 2010 hat eine schwere, überraschend schnell verlaufende Krankheit Dietrich Berke aus dem Kreis seiner Familie, seiner Freunde und Kollegen gerissen. Er hinterlässt spürbare Lücken, denn seine Tätigkeit war vielfältig und verband ihn mit einem großen Kollegenkreis, den er auch durch seine Herzlichkeit und Großzügigkeit gewonnen hatte. Dietrich Berke, geboren am 26. Februar 1938 und aufgewachsen in Castrop-Rauxel, studierte Musikwissenschaft in Kiel und Würzburg, vor allem bei Georg Reichert, und wurde 1967 mit einer Dissertation Studien zur mehrstimmigen französischen Messenkomposition des 14. Jahrhunderts promoviert. Bereits im Herbst desselben Jahres erhielt er ein Forschungsstipendium der DFG für Untersuchungen zu den Textvorlagen der Lieder Schuberts. Zahlreiche Texte dieser Lieder waren damals anonym überliefert, von anderen kannte man zwar den Namen des Dichters, nicht aber die Textquelle, manchmal gar nur die erste Strophe, die der Komponist der Singstimme unterlegt hatte. Berke unternahm dazu weite Reisen: nach Wien, aber auch nach Kremsmünster, nach Brünn (Sammlung Námešt'), nach Oslo, wo der norwegische Sänger Odd Udbye ähnliche Forschungen angestellt und ein umfangreiches Archiv hinterlassen hatte (heute in der Universitätsbibliothek Oslo). Er entdeckte dabei für die Schubert-Forschung (damals hatte auch die Neue Schubert-Ausgabe ihre ersten Bände vorgelegt) bedeutende, bis dahin unbeachtete Abschriftensammlungen. Ein schmaler, jedoch bis heute bedeutsamer Aufsatz fasst die Ergebnisse dieser Reisen zusammen: „Zu einigen anonymen Texten Schubertscher Lieder" (Mf 22, 1969, S. 485-489).

Seine Schubert-Studien hat er auch nach seinem Eintritt in den Bärenreiter-Verlag (1. Juni 1969) unermüdlich weitergeführt, durch die Edition zweier Bände der Neuen Schubert-Ausgabe (Serie III,2, Teile a und b: Mehrstimmige Gesänge für gemischte Stimmen, 1996 und 2006, und Serie III,4: Mehrstimmige Gesänge für gleiche Stimmen ohne Klavierbegleitung, 1974) sowie durch zahlreiche Aufsätze, die sich vorwiegend gleichfalls mit Schuberts mehrstimmigem Vokalwerk befassen (hingewiesen sei vor allem auf 